\title{
No Long-Term Risk for Increased Spread of PNSP in Day-Care Centres by Permitting Carriers to Remain in the Day-Care Centre Group
}

\author{
B. Christenson ${ }^{1}$, B. Henriques Normark ${ }^{2}$, B Ardung ${ }^{1}$, K. Sjöström ${ }^{2}$, G. Eriksson ${ }^{1}$, P. Hartvig ${ }^{3}$ and \\ S.P.E. Sylvan*, \\ ${ }^{I}$ Department of Communicable Disease Control and Prevention, Uppsala County Council, Uppsala, Sweden \\ ${ }^{2}$ Swedish Institute for Infectious Disease Control, Solna, Sweden \\ ${ }^{3}$ Department of Hospital pharmacy, Akademiska Hospital, University of Uppsala, Sweden
}

\begin{abstract}
In a 6-year prospective epidemiological intervention study the spread of pneumococci non-susceptible to penicillin (PNSP) was studied among children attending day-care centres (DCCs). A primary aim was to investigate whether there was a risk for spread of PNSP by permitting children carrying PNSP strains to remain in the DCC. Carriers were followed until they presented negative cultures. The PNSP strains were characterised by serotyping, antibiotic susceptibility testing and clonality. Thirteen children with PNSP, initiated sampling of 321 children, 66 DCC personnel and 149 family members. Twenty-three (7\%) asymptomatic carriers were identified but none among the personnel. The most spread serotype was $6 \mathrm{~B}(47 \%)$, followed by $19 \mathrm{~F}(17 \%)$ and $9 \mathrm{~V}(14 \%)$. The PNSP isolates were resistant to three antibiotics or more $(69 \%)$. The spreading capability and duration of carriage differed between PNSP clones but no long-term risk for increased spread of PNSP in DCCs by carriers was found.
\end{abstract}

\section{INTRODUCTION}

Streptococcus pneumoniae causes common infections ranging from milder upper respiratory tract infections to more severe diseases such as septicemia and meningitis. However, it is also widely spread in the community among healthy children. Carriage rates decrease with age [1] and are particularly high in healthy pre-school children attending day care centres (DCCs), where up to 60-70\% may harbour pneumococci in the nasopharynx [2-8]. Penicillin has been the drug of choice to treat these infections but because of resistance development effective therapy in recent years has been threatened. For decades, the prevalence of pneumococci non-susceptible to penicillin (PNSP) in Sweden has remained low [9-11], whereas in other parts of the world this has become an increasing problem, with up to more than $50 \%$ reduced susceptibility in some countries [12-13]. Prescription of antibiotics varies greatly in Europe, as does the prevalence of resistance to antibiotics [14], with low prevalence in the Nordic countries and high resistance rates in Southern and Central Europe [15]. Furthermore, macrolide resistance in pneumococci has emerged in many parts of the world, even exceeding penicillin resistance in some areas [16]. In addition, multi-resistance poses a new threat that is increasing worldwide and has been observed in more than $50 \%$ of pneumococcal isolates in Hong Kong, Taiwan and South Korea [17].

In Sweden, despite being a low incidence country for antibiotic resistance, multi-resistance is surprisingly high

*Address correspondence to this author at the Department of Communicable Disease Control and Prevention, Uppsala County Council, Dag Hammarskjolds vag 17, SE-751 85 Uppsala, Sweden; Fax: 046185529 01; Email: staffan.sylvan@lul.se among PNSP, up to 40-50\%, suggesting clonal spread of internationally spread clones [11]. The spread of PNSP strains among pre-school children attending DCCs was recognized in Sweden in the beginning of the 1990s, where the problem was especially accentuated in the southern part of the country [18]. Since an explosive increase of PNSP was observed in Iceland [19], a country with a similar high frequency of children attending DCCs, about $75 \%$, Swedish authorities feared that the same increase would emerge in Sweden. Selective pressure of antimicrobial drugs has been suggested as the main factor in the development of resistance [20]. Iceland had the highest prescription of antibiotics among the Nordic countries affecting the spread of PNSP [19]. To reduce the resistance problem in Sweden the National Board of Health and Welfare appointed an expert committee to advise a strategy to limit the spread of PNSP strains among children attending DCCs. The committee recommended intervention involving the screening of the other children in the DCC in connection with the discovery of a child with a PNSP strain. The main strategy for limiting the spread of PNSP strains was to reduce the use of antibiotics and to restrain the children carrying these strains from visiting DCCs until they presented negative cultures.

In the present prospective epidemiological study, performed over a 6-year period, the strategy was to intervene actively in connection with a PNSP diagnose among preschool children attending DCCs and to permit healthy children carrying a PNSP strain to remain at the DCC. A main question was whether there was an increased risk in the spread of PNSP if children carrying PNSP were allowed to stay in the DCC group. A reported PNSP at a DCC initiated sampling of the children at the same DCC. Clinical information about prior and current infections was collected and personnel at the DCCs as well as family members in close 
contact with the child carrying PNSP, were sampled. The duration of carriage of PNSP was estimated by repeated sampling of the children carrying PNSP at the DCCs. To study the spread of pneumococcal clones, isolates recovered were characterized by antibiotic susceptibility testing, serotyping and molecular typing using pulsed-field electrophoresis (PFGE) and multi-locus sequencing typing (MLST).

\section{MATERIALS AND METHODOLOGY}

\section{Study Area}

Since 1996, all strains of S. pneumoniae have been routinely screened for penicillin resistance in Uppsala County (a population of nearly 300,000 ), a region close to the Stockholm urban area. All PNSP strains (MIC penicillin G, $\geq 0.5$ $\mathrm{mg} / \mathrm{L}$ ) have been reported from microbiology laboratories to the Regional Centre of Communicable Disease Control. Between 1996 and 2001, totally 88 PNSP isolates were reported in Uppsala County (Table 1). During this period, a decreasing prevalence of PNSP strains was observed: 28 such strains were reported in 1996 as compared with only 8 each year in 2000 and 2001. All PSNP strains reported from preschool children attending DCCs led to nasopharyngeal sampling of children (after parental consent) and personnel attending the same DCC group.

\section{Sampled Population}

During the study period 1996-2001, 36 of the 88 isolates reported came from children attending DCCs, of which 13 isolates initiated nasopharyngeal sampling of 10 DCCs and 3 private day-care (PDC) homes.The remaining 23 isolates were recovered from the sampling of these day-care/homes. Totally, nasopharyngeal swabs were collected from 321 children, 66 personnel and 149 family members and close contacts (Table 2). Children without symptoms were not given any antimicrobial drug. PNSP carriers were, in most cases, cultured every second week. In two DCCs and in one PDC home (DCCs nos. 1, 2 and 7), 58 children and 15 daycare personnel were additionally sampled after the first sampling. In connection with the sampling with nasopharyngeal swabs the parents were asked to complete a questionnaire about current and prior infections and whether they regarded their child as often being infected or more susceptible to infections as compared with other children. They were also asked if their child had otitis media before one year of age. Healthy children discovered to be carriers of PNSP strains were not restrained from attending the DCC.

\section{Drug Susceptibility Testing.}

All isolates were tested for drug susceptibility using the standard oxacillin-disc diffusion method [21]. Strains with reduced sensitivity to penicillin (MIC > 0.1) were tested for susceptibility to erythromycin, chloramphenicol, trimethoprim-sulphamethoxazole, tetracycline and clindamycine by using the disk diffusion method according to the Swedish Reference Group for Antibiotics (SRGA). Inhibition zone diameters were read to the nearest millimetre and interpreted according to SRGA guidelines. All procedures were performed according to routine protocols approved by the Swedish Board for Accreditation and Conformity Assessment and recommendations from the Swedish Institute for Infectious Disease Control [22].

\section{Serotyping of Pneumococcal Isolates}

Twenty-five isolates were available for serotyping. Pneumococcal strains were identified by optochin sensitivity. All strains were serotyped at the Swedish Institute for Infectious Disease Control by gel diffusion, using 46 typeor group-specific serum samples [23] obtained from the World Health Organization (WHO) Collaborating Centre for Reference and Research on Pneumococci at Statens Seruminstitut (Copenhagen, Denmark). Isolates that could not be typed or that belonged to a group that included several types were examined by capsular reaction test (Quellung test) and/or gel diffusion with type-specific antisera [24] from the WHO collaboration centre.

\section{Molecular Typing}

Two molecular typing methods were used for characterizing the clinical isolates, namely PFGE and MLST. Totally, 13 isolates were analysed by using these two methods.

\section{PFGE-Pulsed Field Gel Electrophoresis}

The PFGE method was adapted from the procedure depicted by Hermans et al. [25] as described previously [26]. The gel was analysed by using the programme BioNumerics version 2.5 (Applied Math, 1998), with the Dice coefficient and UPGMA dendrogram type. The optimization setting was $1.50 \%$ and position tolerance was $1.00 \%$. The clones were defined using the criteria described by Tenover et al. [27], i.e. isolates that differed with $\leq 3$ bands were considered closely related.

\section{MLST - Multi Locus Sequence Typing}

This method was adapted from the procedure depicted by Enright et al. [28]. Chromosomal DNA from pneumococcal

Table 1. PNSP Isolates Reported in Uppsala County, 1996 - 2001.

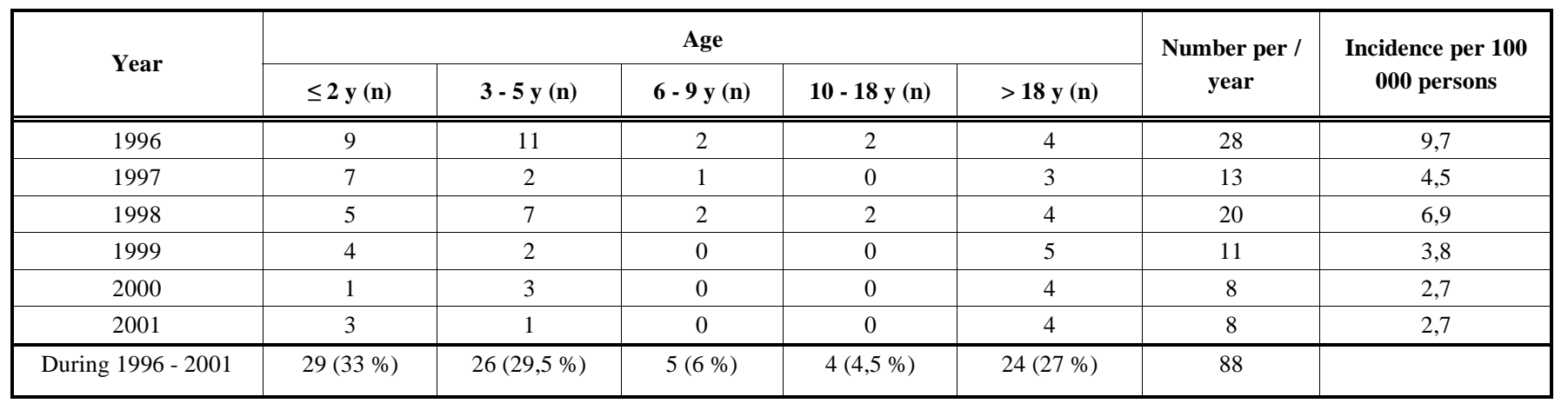


Table 2. Characterization of Carriage of PNSP Isolates in the 13 DCCs / Private Day-Care Homes Included in the Study

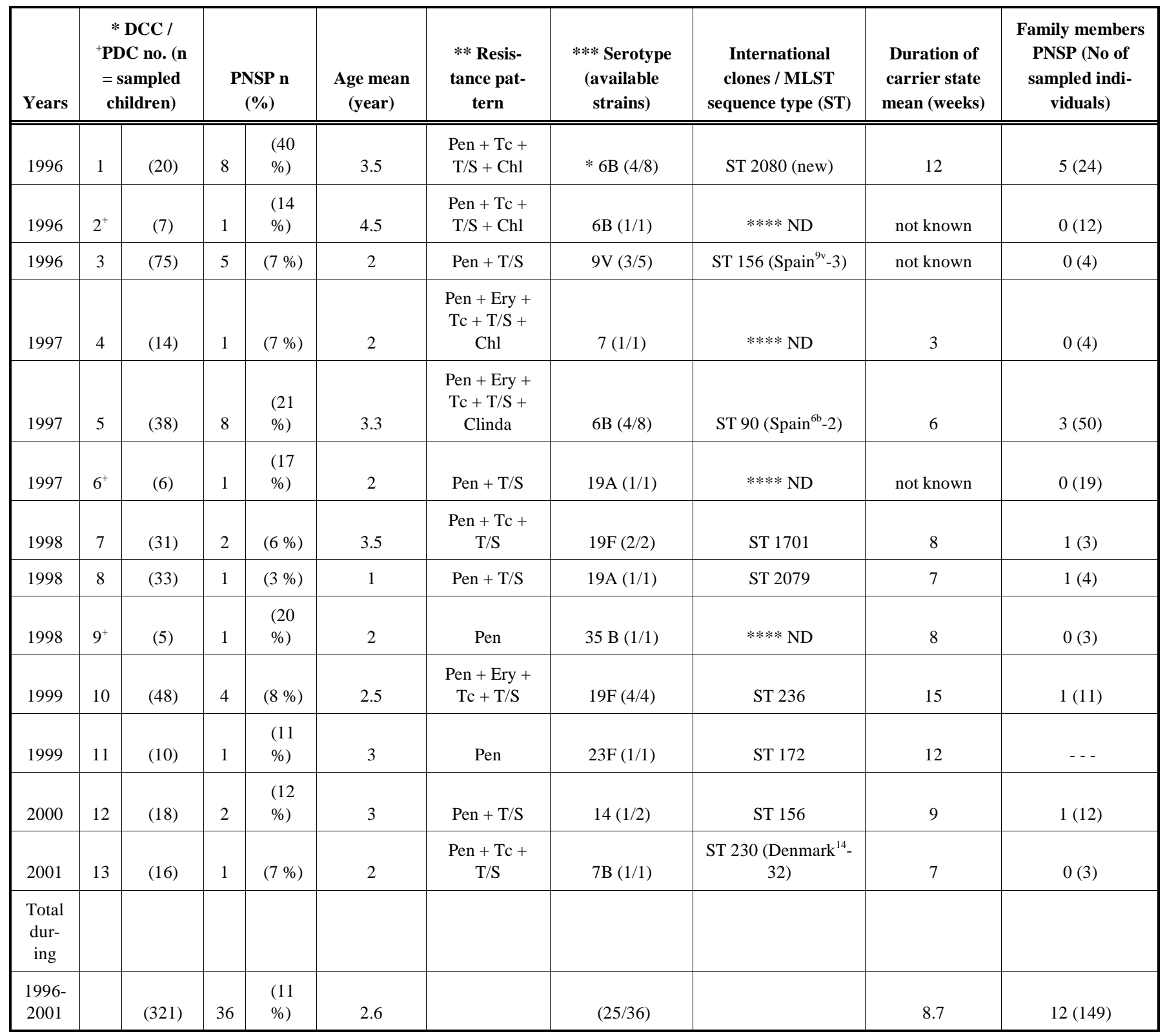

$*$ DCC $/$ PDC $=$ Day-care-center $/{ }^{+}$private day care-home.

** Resistance from all PNSP isolates Pen, Bensylpenicillin; Tc, tetracycline; T/S; trimethoprim/sulphamethoxazole; Chl, chloramphenicol; Ery; erythromycin, Clinda; clindamycine. *** 25 out of 36 PNSP isolates were serotyped.

$* * * * \mathrm{ND}=$ not done.

isolates were subjected to sequencing of seven different housekeeping genes (aroE, gdh, gki, recP, spi, xpt, and $d d l$ ) using an Applied Biosystems Prism 3700 automated sequencer (PE Applied Biosystems). The sequences obtained were submitted to the MLST database on the Internet, http://www.mlst.net, where they were assigned alleles at each locus and a sequence type (ST).

\section{RESULTS}

\section{Carriage of PNSP and Symptoms at Sampling Among Children at the DCCs}

Of the 321 children attending DCCs and PDC homes, 36 $(11 \%)$ were carriers of PNSP isolates (Table 2), while none of the 66 personnel had pneumococci in the nasopharynx. Of the family members, 12 of 149 sampled (8\%) had PNSP strains. The frequency of PNSP varied among the DCCs from as low as $3 \%$ to as high as $40 \%$. Further, a reduction was observed with time in the number of PNSP cases reported from DCCs in Uppsala County (from 14 cases in 1996 to 1 case in 2001).

Of the 13 children initiating sampling of the children at the DCCs, the most common reason for a nasopharyngeal culture was upper respiratory tract infections without otitis media (43\%), followed by otitis media (29\%). Most of the children carrying PNSP strains were healthy when sampled at the DCCs, however, the questionnaires revealed that $30 \%$ had a current infection, defined as a slight cold, compared with $28 \%$ of the children without a PNSP strain (Table 3). 
Table 3. Current and Prior Infections in Children Carrying PNSP Strains as Compared to Non-Carries

\begin{tabular}{|c|c|c|}
\hline & Children with PNSP n = 23 & Children without PNSP n = 279 \\
\hline \hline Current infection & $7(30 \%)$ & $79(28 \%)$ \\
\hline Otitis media $<1$ year & $7(30 \%)$ & $72(26 \%)$ \\
\hline Prior pneumonia & $2(9 \%)$ & $32(11 \%)$ \\
\hline Susceptible to infections * & $4(17 \%)$ & $49(18 \%)$ \\
\hline
\end{tabular}

* Often infected or more susceptible to infections, compared to other children.

There were no significant differences in prior otitis media, pneumonia or susceptibility to infections between carriers of the PNSP strains and the remaining children.

\section{Duration of Carriage of PNSP}

Repeated sampling was performed on all children and personnel at two DCCs (DCC 1 in 1996 and DCC 7 in 1998) as well as in one family day-care (DCC 2).

In the first sampling of DCC 1, 5 of 20 children but none of the 4 personnel carried PNSP strains; all serotyped were serotype 6B. The second sampling was done after 8 weeks. All but one of the carriers were now negative and three other children had become carriers with the same PNSP strain as assessed by serotyping and susceptibility testing. After a further 8 weeks, a third sampling was performed of all children and personnel. None of the children carried PNSP strains, except for the one child that became a carrier for 36 weeks. Screening was also performed at a PDC home (DCC 2 ), with close contact with DCC 1 through siblings and playmates, and therefore one additional carrier was found among the children.

Of the 31 children sampled in DCC 7, only one carried a PNSP strain of serotype 19F. Repeated sampling 8 weeks later of all the children showed that none had a PNSP strain.

All carriers of PNSP isolates were sampled mostly every second week to estimate the duration of carriage (Table 2). The mean duration of carriage varied between 3 and 15 weeks in the different DCCs and in most cases the carriage spontaneously disappeared after 6-12 weeks.

In two cases carrying PNSP of isolate serotypes $19 \mathrm{~F}$ and $6 \mathrm{~B}$ the carrier state was 28 and 36 weeks, respectively.

\section{Spread of Internationally Recognized PNSP Clones}

Only one serotype and one antibiotic susceptibility pattern was found among the PNSP isolates at each DCC (Table 2), suggesting clonality. Totally, seven serotype/groups were found among PNSP in decreasing order among the children at the DCCs: 6B (47\%), 19F (17\%), 9V (14\%), and 7,14 and $19 \mathrm{~A}(5 \%)$ and $23 \mathrm{~F}$ and $35 \mathrm{~B}(3 \%)$. The most widely spread serotype, $6 \mathrm{~B}$, potentially spread to 25 children and family members (Table 2). The majority of the PNSP isolates $(58 \%)$ were intermediately susceptible to penicillin (MIC penicillin $\mathrm{G} \geq 0.5 \mathrm{mg} / \mathrm{L}$ and $\leq 1 \mathrm{mg} / \mathrm{L}$ ). Only one child (from a family-day-care home ,1997), had a PNSP with a MIC value of $\geq 2 \mathrm{mg} / \mathrm{L}$

A majority (69\%) of the PNSP isolates were multiresistant, which were of serotypes/groups $6 \mathrm{~B}, 7$ and 19F. The most common resistance pattern was to trimethoprim/ sulphamethoxazole (94\%), found in all except two isolates, 35B and $23 \mathrm{~F}$, both with reduced susceptibility to penicillin alone. Among the type 6B isolates all 14 PNSP isolates from 1996 had the same resistance pattern, including resistance to tetracycline, trimetoprim/sulfametoxazole and chloramphenicol, whereas the 6B isolates from 1997 were sensitive to tetracycline but had additional resistance to erythromycin and clindamycin.

Molecular typing with PFGE and MLST revealed that the isolates of 6B from 1996 and 1997 were closely related and belonged to the same clonal cluster by MLST (ST 2080 and ST 90). ST 2080 is a new single locus variant of the ST 90 clone, which is the internationally spread clone Spain ${ }^{6 \mathrm{~B}}-2$, also found in Australia, the USA, Iceland, Taiwan and Italy [29].

The second most common serotype was $19 \mathrm{~F}$, which was found in two DCCs. The isolates belonged to two different clones ST 1701 in DCC 7 and ST 236 in DCC 10. These two clones had differences in their susceptibility patterns (Table 2). ST 1701 isolates have previously been found in India (serotype 7C) and ST 236 is a major multi-resistant clone found in the UK, the USA, Australia, Vietnam and China.

The internationally spread clone Spain ${ }^{9 \mathrm{~V}}-3$ with ST 156 was found among pneumococcal isolates from two DCCs (DCC 3 and 12). However, the isolates were of different serotypes, $9 \mathrm{~V}$ and 14, resulting from a capsular switch. ST 156 has been found in several countries, including Israel, Denmark, Canada and Brazil.

\section{DISCUSSION}

This 6-year prospective epidemiological study focused on the carriage and spread of PNSP strains among pre-school children attending DCCs in Sweden, a known low PNSP incidence area.

The study had an intervention approach and was an attempt to estimate the risk for spread of PNSP strains in children allowed to remain in the DCC group. Previously, a study was performed in 2002 investigating the effect of different strategies for limiting the spread of PNSP in Swedish DCCs [30], including active intervention by restraining carriers from attending DCCs. However, because of sample sizes, the study could not convincingly demonstrate the effects of the intervention program compared with keeping the carriers in the DCC groups. In our study we found that between 3 and $40 \%$ of the children at the DCCs/PDCs carried PNSP, which could be genetically related. However, when repeated sampling was performed in two DCCs, where a 19F 
and a 6B PNSP strain was circulating respectively, the $19 \mathrm{~F}$ strain was shown to have disappeared 8 weeks later, whereas the 6B strain showed higher spreading capability and new children had become carriers at the 8 -week sampling. Because all PNSP carriers were sampled mostly every second week, we could make an estimation of the duration of carriage. It was found to differ between 2 and 36 weeks, with the longest carriage rate for a $6 \mathrm{~B}$ strain. These data suggest that the spreading capability and duration of carriage may differ between clones and serotypes. They also imply that virulence attributes such as the capsule and other factors (e.g., the recently described pilus like structure) [31], may influence the capacity of different PNSP clones in their spread among children at DCCs.

However, in most cases the carriage disappeared spontaneously after 6-12 weeks suggesting that either a protective immune response has been elicited or that the PNSP clones have been out-competed by more fit susceptible pneumococcal strains.

It is notable that even in a country like Sweden with a low incidence of PNSP we find that a high proportion of the PNSP isolates carry additional resistances, where as much as $69 \%$ of the PNSP isolates were multi-resistant. This observation emphasises that the spread of resistance is largely attributed to the spread of international PNSP clones. The Spain $^{6 \mathrm{~B}}-2$ clone found here has previously been observed to spread to Iceland and increased the resistance rates to penicillin in this country during the 1990s [19]. The Spain ${ }^{9 \mathrm{~V}}-3$ clone, found as type $9 \mathrm{~V}$ and 14 in this study, is spreading around the world and, for example, is the most common clone in the Czech Republic [32]. Isolates of type 9Vand 14 are also the most commonly found serotypes in Sweden at the moment [30] and it remains to be shown whether this clone is prominent among the Swedish type 9V and 14 PNSP as well. In an earlier study we demonstrated the spread of an international clone of type 35B in Sweden [7]. In the present study we found one child at a PDC home carrying a serotype 35B. Unfortunately, this strain was not available for MLST typing. The most common serotypes in this study were $6 \mathrm{~B}$, $19 \mathrm{~F}, 9 \mathrm{~V}, 7,19 \mathrm{~A}, 23 \mathrm{~F}$, and 35B, a finding in close agreement with other studies [33,34]. Most of the serotypes are included in the 7-valent conjugated vaccine, with the exception of serotypes 35B, 19A and 7/7B, which were found in four DCCs/PDCs.

During the study period, the incidence of isolated PNSP strains decreased in the study area (Table 1). In the first two years (i.e. 1996 and 1997) 5 children were reported from DCCs which initiated sampling of 160 children, and a further 19 children were found with a PNSP strain (12\%). In the following 4 years (i.e. 1998-2001) 7 children with a PNSP strain were reported from DCCs and 161 children were sampled at DCCs where 5 carriers were found (3\%). One reason for the decreasing incidence observed might be ascribed to the reduction in the number of nasopharyngeal samples taken during the same period. Another possible reason might be a more restricted use of antimicrobial drugs. In Sweden, as in many other countries, the awareness of an association between resistance development [20] and antibiotic usage has increased; in fact, during the study period, a major decrease of antibiotic consumption was observed [35,36]. However, at the same time, an increase was noted in the proportion of
PNSP with additional resistance [11] and there were hardly any correlations between antibiotic sales and PNSP rates during the same period in Sweden [11]. Further, better hygiene routines have attracted more attention lately, which may also be a contributing factor to the decreasing incidence. One possibility is also that the most successful clone of type $6 \mathrm{~B}$, which was not observed after 1997, has been outcompeted by less transmissible clones or by immune responses evoked in the population.

The spread of PNSP isolates was not restricted to DCC attendance because eight siblings and four parents were infected with the same PNSP strain as the child attending a DCC. However, no PNSP strain was found among the personnel at the DCCs. This finding suggests that the spread of PNSP isolates in the close family group is as important as in the DCCs group, and therefore restraining children from attending DCCs will not affect other contacts. Moreover, in accordance with what others have found, our data suggest that each DCC functions as an epidemiologic unit $[37,38]$.

\section{CONCLUSION}

In conclusion, we did not find any long-term risk in this study for increased spread of PNSP in DCCs by letting carriers remain in the DCC group. However, we found that the spreading capability may vary between different PNSP clones, indicating that different intervention strategies are required depending on which clones are circulating in the community.

\section{ACKNOWLEDGMENT}

This study was partly supported by the project PREVIS within EU's $6^{\text {th }}$ framework programme, the Swedish Royal Academy of Sciences, Torsten and Ragnar Söderbergs foundation and the Swedish Research Council.

\section{REFERENCES}

[1] Christenson, B.; Sylvan, S.P.E.; Noreen, B. Scand. J. Dis., 1997, $29,555$.

[2] Lopez, B.; Cima, M.D.; Vasquez, F.; Fenoll, A.; Gutierrez, J.; Fidalgo, C.; Caicoya, M.; Mendez, F.J. Eur. J Clin. Microbiol. Infect. Dis., 1999, 18, 771 .

[3] Duchin, J.S.; Breiman, R.F.; Diamond, A.; Lipman, H.B.; Block, S.L.; Hedrik, J.A.; Finger, R.; Elliott, J.A. Pediatr. Infect. Dis. J., 1995, 14, 745.

[4] Breiman, R.F.; Butler, J.C.; Tenover, F.C.; Elliott, J.A.; Facklam, R.R. JAMA, 1994, 271, 1831.

[5] Jernigan, D.B.; Cetron, M.S.; Breiman, R.F. JAMA, 1996, 275, 206.

[6] Syrjanen, R.K.; Kilpi, T.M.; Kaijalainen, T.H.; Herva, E.E.; Takala, A.K. J. Infect. Dis., 2001, 184, 451.

[7] Henriques Normark, B.; Christenson, B.; Sandgren, A.; Noreen, B.; Sylvan, S.P.E.; Burman, L.G.; Olsson-Liljequist, B. Microb. Drug Resist., 2003, 9, 337.

[8] Nunes, S.; Sá-Leao, R.; Carrico, J.; Alves, C.R.; Mato, R.; Brito Avo, A.; Almeida, J.S.; Santos Sanches, I.; de Lancastre, H. J. CLIN Microbiol., 2005, 43, 1285.

[9] Christenson, B.; Sylvan, S.P.E.; Noreen, B. J. Infect. Dis., 1998, $37,9$.

[10] Borres, M.P.; Alestig, K.; Krantz, I.; Larsson, P., Norvenius, G.; Stenqvist, K. J. Infection, 2000, 40, 141.

[11] Högberg, L.; Ekdahl, K.; Sjöström, K.; Olsson-Liljequist, B.; Walder, M.; Melander, E.; Ringberg, H.; Henriques Normark, B. Microb. Drug Resist., 2006, 12, 16.

[12] Baquero, F.; Garcia-Rodriguex, J.A.; Garcia de Lomas, J.; Aguilar, L. Antimicrob. Agents Chemother., 1999, 43, 357.

[13] Yagupsky, P.; Porat, N.; Fraser, D.; Prajgrod, F.; Merires, M.; McGee, L.; Klugman, K.P.; Dagan, R. J. Infect. Dis., 1998, 177, 1003. 
[14] Goossens H, Sprenger M. BMJ,1998, 7159, 654.

[15] Bronzwaer, S.L.; Cars, O.; Buchholz, U.; Mölstad, S.; Goettsch, W.; Veldhuijzen, I.K.; Kool, J.L.; Sprenger, M.J.; Degener, J.E. Emerg. Infect. Dis., 2000, 3, 278.

[16] Felmingham, D.; Reinert, R.R.; Hirakata, Y.; Rodloff, A. J Antimicro Chemother., 2002, 50(Suppl), 25.

[17] Felmingham, D. Chemotherapy, 2004, 50(Suppl 1), 3.

[18] Ekdahl, K.; Kamme, C. Scand. J. Infect. Dis., 1994, 26, 301.

[19] Kristinsson, K.G.; Hjalmarsdottir, M.A.; Steingrimsson, O. Lancet, 1992, 339, 1606.

[20] Goossens, H.; Ferech, M.; Stichele, R.V.; Elseviers, M. Lancet, $\mathbf{2 0 0 5}, 365,579$.

[21] Swedish Reference Group for Antibiotic (SRGA). Reference and Method Book. Swedish Institute for Infectious Disease Control, 1990.

[22] National Committee for Clinical Laboratory Standards. Performance standards for antimicrobial disk susceptibility tests. Fourth edition, Approved standard. NCCLS Document M2-A4. Villanova, $\mathrm{Pa}$; NCCLS, 1990.

[23] Halbert, S.P.; Swick, L.; Sonn, C. J. Exp. Med., 1955, 101, 557.

[24] Lund, E.; Henrichsen, J. In Methods in microbiology, Ed.; Bergan, T. and Norris, J.R. Academic Press, New York, 1978; vol. 12, pp. 241-262.

[25] Hermans, P.W.; Sluuter, M.; Hoogenboezem, T.; Heersma, H.; van Balkum, A.; de Groot, R. J. Clin. Microbiol., 1995, 33, 1606.

[26] Sjöström, K.; Spindler, C.; Örtqvist, Å.; Kalin, M.; Sandgren, A.; Kuhlman-Berenzon, S.; Henriques Normark, B. Clin. Infect. Dis., 2006, 15,451 .

[27] Tenover, F.C.; Swaminathan, B. J. Clin. Microbiol., 1995, 33, 2233.

[28] Enright, M.C.; Spratt, B.G. Microbiology, 1998, 144, 3049.
[29] McGee, L.; McDougal, L.; Zhou, J.; Spratt, B.G.; Tenover, F.C.; George, R.; Hackenbeck, R.; Hryniewicz, W.; Lefevre, J.C.; Tomasz, A.; Klugman, K.P. J. Clin. Microbiol., 2001, 39, 2565.

[30] Högberg, L.; Henriques Normark, B.; Ringber, H.; Stenqvist, K.; Fredlund, H.; Geli, P.; Gabowska, K.; Melander, E.; Laurell, M.; Åhren, C.; Törnqvist, E.; Fält, R.; Höglund, D.; Möllerberg, G.; Ekdahl, K. Scand. J. Infect. Dis., 2004, 36, 629.

[31] Barrocchi, M.; Ries, J.; Zogaj, X.; Albiger, B.; Kanth, A.; Dahlberg, S.; Moschioni, M.; Masignani, V.; Hultenby, K.; von Euler, A.; Holden, D.W.; Covacci, A.; Normark, S.; Rappuoli, R.; Henriques Normark, B. Proc. Natl. Acad. Sci. USA, 2006, 103, 2857.

[32] Zemlickova, H.; Melter, O.; Urbaskova, P. J. Med. Microbiol., 2006, 55, 437.

[33] Henriques, B.; Kalin, M.; Örtqvist, Å.; Olsson-Liljequist, B.; Svensson, S.B.; Marrie, T.; Mufson, M.; Woodhead, M.; Torres, A.; Källenius, G. J. infect. Dis., 2000, 182, 833.

[34] Melander, E.; Ekdahl, K.; Hansson, H.B.; Kamme, C.; Laurell, M.; Nilsson, P.; Persson, K.; Söderström, M.; Mölstad, S. Mikrob. Drug Resist., 1998, 4, 71 .

[35] Melander, E.; Ekdahl, K.; Jonsson, G.; Molstad, S. Pediatr. Infect. Dis. J., 2000, 19, 1172.

[36] Cars, O.; Ekdahl, K. SWEDDRES 2003 14A report on Swedish antibiotic utilisation and resistance in human medicine. STRAMA and Swedish Institute for Infectious Disease Control (SMI), 2004

[37] Sá-Leáo, R.; Tomasz, A.; Sanches, I.S.; Nunes, S.; Alves, R.; Brito-Avo, A.; Saldanha, J.; Kristinsson, K.G.; de Lancastre, H. J. Clin. Microb., 2000, 38, 4137.

[38] Sa-Leao, R.; Tomasz, A.; Sanches, I.S.; Brito-Avo, A.; Vilhelmson, S.E.; Kristinsson, K.G.; de Lancastre, H. J. Infect. Dis., 2000, 182, 1153. 